Monatsschr Kinderheilkd 2019 $\cdot$ 167:545 https://doi.org/10.1007/s00112-019-0701-9 Online publiziert: 29. April 2019

(c) Springer Medizin Verlag GmbH, ein Teil von Springer Nature 2019

\section{Redaktion}

G. Hansen, Hannover

R. Kerbl, Leoben

F. Zepp, Mainz

\section{Leserbrief zu}

Christen H-J, Eiffert H (2019) Neuroborreliose im Kindesalter. Monatsschr Kinderheilkd 167: 67-79. https://doi.org/10.1007/s00112-0180627-7

\section{Erwiderung}

Christen H-J, Eiffert H (2019) Stellenwert der Doxycyclinprophylaxe und Sklerodermierisiko nach Zeckenstich. https://doi.org/10.1007/ s00112-019-0702-8

Zur Publikation „Neuroborreliose im Kindesalter" von H.-J. Christen und H. Eiffert in der Monatsschrift Kinderheilkunde 01/2019 folgende zwei kurze Fragen: Die erste bezieht sich auf ihre Empfehlung, keine antibiotische Prophylaxe nach Zeckenstich durchzuführen; die zweite auf ihre Ausführung, dass es für eine "chronische Borreliose" keine fundierten Beläge gäbe.

\section{Antibiotikaprophylaxe}

Die Leitlinie der Infectious Diseases Society of America (IDSA, [1]) für Borreliose zitiert Untersuchungen, nach denen sich mit zunehmender Anheftungsdauer der Zecke das Risiko für eine Borreliose deutlich erhöht. Der Leitlinie sind Fotos beigefügt, auf denen man anhand der Größe der vollgesogenen Zecken (Nymphen und adulte Tiere) die Anheftungsdauer abschätzen kann (allerdings „Pi mal Daumen“). Folgende Empfehlung wird gegeben: wenn a) eine mehr als $36 \mathrm{~h}$ festsitzende Nymphe oder adulte Zecke entdeckt wird, b) man binnen $72 \mathrm{~h}$ nach Entfernung mit einer

\title{
Romanus Röhnelt
}

Neonatologe, Asthmatrainer, fachgebundene humangenetische Beratung, Qualitätszirkel der Kinder- und Jugendärzte im Kreis WAF, Warendorf, Deutschland

\section{Chronische Borreliose als Ursache einer Morphea?}

\section{Antibiotische Prophylaxe nach Zeckenstich?}

antibiotischen Therapie starten könnte, und wenn c) mindestens jede 5. Zecke der Gegend Borrelien-positiv ist (wie z.B. in Deutschland), dann sollte eine Einzeldosis Doxycyclin gegeben werden, sofern d) keine Kontraindikationen dafür bestehen.

\section{Chronische Borreliose}

Offensichtlich gibt es in der Kinderdermatologie Hinweise auf eine immunologisch nichtbewältigte Borrelieninfektion, so bei jugendlichen Morphea-Patienten [2]. Ich selbst betreue eine Morphea-Patientin mit inzwischen ausgedehnter kutaner Sklerodermie, die Jahre nach einem Erythema migrans und persistierenden Borrelienantikörpern an einer Hemiatrophia facei leidet. Sie wurde von der Universitätshautklinik nun aufgrund der Annahme einer persistierenden Immunreaktion gegen Borrelien zunächst 14 Tage mit Ceftriaxon $\left(\right.$ Rocephin $^{\circledR}$ ) behandelt.

Könnten Sie diese Differenzen aus Ihrer Sicht kommentieren?

\section{Korrespondenzadresse}

\section{Dr. Romanus Röhnelt}

Neonatologe, Asthmatrainer, fachgebundene humangenetische Beratung, Qualitätszirkel der Kinder- und Jugendärzte im Kreis WAF Am Krankenhaus 4, 48231 Warendorf, Deutschland

dr.r.roehnelt@t-online.de

Interessenkonflikt. R. Röhnelt gibt an, dass kein Interessenkonflikt besteht.

\section{Literatur}

1. Wormser GP, Dattwyler RJ, Shapiro ED, Halperin JJ, Steere AC, Klempner MS, Krause PJ et al (2006) The clinical assessment, treatment, and prevention of Lyme disease, human granulocytic anaplasmosis, and babesiosis: clinical practice guidelines by the Infectious Diseases Society of America. Clin Infect Dis 43(9):1089-1134. https://doi.org/10.1086/ 508667

2. Prinz JC, Kutasi Z, Weisenseel P, Pótó L, Battyáni Z, Ruzicka T (2009) „Borrelia-associated earlyonset morphea": a particular type of scleroderma in childhood and adolescence with high titer antinuclear antibodies? Results of a cohortanalysis and presentation of three cases. J Am Acad Dermatol 60:248-255 